\title{
Design of a novel zig-zag 192+192 Row Column Addressed Array Transducer: A simulation study.
}

Schou, Mikkel; Havreland, Andreas Spandet; Engholm, Mathias; Stuart, Matthias Bo; Thomsen, Erik Vilain; Jensen, Jørgen Arendt

\section{Published in:}

Proceedings of 2018 IEEE International Ultrasonics Symposium

Link to article, DOI:

10.1109/ULTSYM.2018.8579941

Publication date:

2018

Document Version

Peer reviewed version

Link back to DTU Orbit

Citation (APA):

Schou, M., Havreland, A. S., Engholm, M., Stuart, M. B., Thomsen, E. V., \& Jensen, J. A. (2018). Design of a novel zig-zag 192+192 Row Column Addressed Array Transducer: A simulation study. In Proceedings of 2018 IEEE International Ultrasonics Symposium [8579941] IEEE. https://doi.org/10.1109/ULTSYM.2018.8579941

\section{General rights}

Copyright and moral rights for the publications made accessible in the public portal are retained by the authors and/or other copyright owners and it is a condition of accessing publications that users recognise and abide by the legal requirements associated with these rights.

- Users may download and print one copy of any publication from the public portal for the purpose of private study or research.

- You may not further distribute the material or use it for any profit-making activity or commercial gain

- You may freely distribute the URL identifying the publication in the public portal 


\title{
Design of a novel zig-zag 192+192 Row Column Addressed Array Transducer: A simulation study.
}

\author{
Mikkel Schou ${ }^{1}$, Andreas Spandet Havreland ${ }^{2}$, Mathias Engholm ${ }^{2}$, Matthias Bo Stuart ${ }^{1}$, \\ Erik Vilain Thomsen ${ }^{2}$ and Jørgen Arendt Jensen ${ }^{1}$ \\ ${ }^{1}$ Center for Fast Ultrasound Imaging, Dept. of Elec. Eng., Bldg. 349. ${ }^{2}$ Department of Micro and Nanotechnology, \\ Technical University of Denmark, 2800 Kgs. Lyngby, Denmark
}

\begin{abstract}
Super resolution imaging (SRI) can benefit greatly from full 3D imaging to compensate for vessel structures moving out of the image plane. Row Column Addressed (RCA) arrays can provide such 3D imaging with low-complexity probe design. A RCA probe is being designed for a rat kidney with $192+$ 192 elements to ensure low sidelobes and an imaging volume of $15 \times 15 \times 15 \mathrm{~mm}^{3}$. The design space of such a transducer is investigated with this in mind. Capacitive micromachined ultrasonic transducer (CMUT) technology allows for new geometric shapes, including a near kerf-less zig-zag interwoven structure, which provides flexibility in the design process of new RCA. This work compares the image quality of a straight element RCA array with an RCA array with an interwoven zig-zag structure by simulations, to assess the image quality of a zigzag structured RCA. The Point Spread Function (PSF) showed symmetry in both the azimuth and elevation direction. Both the straight and interwoven design had a lateral Full Width Half Maximum (FWHM) of approximately $0.6 \lambda$, close to the ideal FWHM without apodization. The interwoven design showed a slight contrast loss over the straight desig, which was quantified with the Cystic Resolution (CR). The CR at $20 \mathrm{~dB}$ for the straight design was $1.3 \lambda$, compared to $1.4 \lambda$ of the interwoven design. The two designs had comparable PSF metrics. The interwoven zig-zag structure is therefore a viable solution to meet the requirements of the rodent experiments and provides a new level of design flexibility for manufacturing RCA transducer arrays.
\end{abstract}

\section{INTRODUCTION}

Super Resolution Imaging (SRI) can become an invaluable tool for understanding and diagnosing various disease processes that influences the microvascular blood flow, such as cancer, stroke, and arteriosclerosis [1]. SRI combines the use of microbubbles, a long known contrast agent used in medical ultrasound, with precise tracking of their temporal movement through a series of ultrasound frames to enhance the spatial resolution beyond the diffraction limit of the ultrasound system. The 2-D SRI method in its current state is prone to errors. This include movement artifacts from tissue, respiration and movement of the transducer probe itself [2], which complicates the microbubble tracking even more. The errors due to motion are often solved by fixating the subject and the transducer probe, which is unrealistic in a real clinical setting.

Another suspected source of error is vessel structures moving in and out of the image plane the in elevation plane [2]. 2-D ultrasound systems often uses a 1-D array, which have a broad beam in elevation, thereby receiving echoes from microbubble outside the image plane [3]. 3-D ultrasound can capture a full
3-D volume and is in principle not limited to a single plane in elevation. However implementation of 3-D ultrasound have proven itself difficult. 3-D imaging techniques can in general be performed using either one of two methods. Either using a translating 1-D array or a 2-D array. 2-D arrays can further be divided into fully populated arrays, using microbeamformers, or as Row-Column Addressed Arrays (RCA) [4]. Methods such as translating the ultrasound probe from emission to emission have shown 3-D volumes, but when looking at vessel structures at around $2-20 \mu \mathrm{m}$ in size [3], translation must be performed in a highly precise manner. Combining this with the long acquisition time required for SRI makes the method highly impractical for SRI. A matrix probe has shown great resolution in 3-D, this however requires $N^{2}$ number of connections where $N$ is the number of elements in each dimension of the 2-D array, or by microbeamforming which puts high demand on the front end electronics [5]. RCA have shown potential for lower production cost and the use of fewer connections allows for a simpler setup.

An in-vivo measurement of a rat kidney is in development to test the feasibility of 3-D SRI. The dimension of the rat kidney requires an acoustical window of approximately 15 by $15 \mathrm{~mm}$, which is assumed to be the desired dimensions of the RCA probe [6]. The intended technology for the probe is Capacitative Micromachined Ultrasonic Transducer (CMUT) elements, which allows for manufacturing of new structures. The channel count of $192+192$ combined with a high frequency necessitates flexibility in the manufacturing process of the CMUT array. For this reason a new kerf-less interwoven zig-zag structure is introduced. The new interwoven zig-zag RCA design allows for different implementation method and is therefore investigated here. In this work we present a simulation model and results of a $192+192,8 \mathrm{MHz}$ interwoven RCA design, to evaluate whether it carries a penalty in imaging performance compared to a traditional geometry.

\section{Methods}

\section{A. Row Column addressed arrays}

The standard RCA probe is constructed as a matrix array where only the rows and columns can be addressed, limiting the number of connections to $2 N$. A cutout of the straight RCA is visualized in Fig. 1 to the left. The row and columns of the RCA are connected such that a single element is comprised of $M$ cells, and $N=M$. For the interwoven design two 

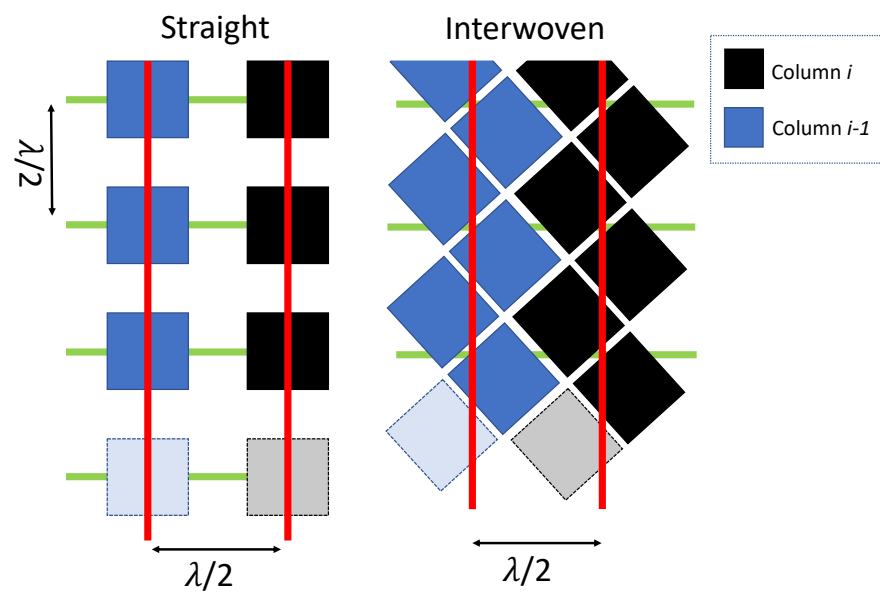

Fig. 1: Simulation setup. To the left the straight element RCA is visualized, the cells have $\lambda / 2$ spacing which matches the inter-element spacing. To the right the intervowen structure is visualized. The red lines symbolizes the assumed acoustic center of the column elements, the green likewise for the rows.

cells are acting as a single unit, by arranging the dual cells in a zig-zag like structure, one element is then comprised of $2 M$ cells. The interwoven design gives an overlapping region between two neighboring elements causing an almost kerfless like structure as seen to the right in Fig. 1. A larger aperture size provides a method of maintaining a constant resolution in a deeper region, and it has been observed that the resolution and contrast of RCA transducers scales with number of channels [7]. The RCA array offers a linear scale of number of channels as oppose to the matrix probe making it ideal for larger aperture sizes. Grating lobes arises when the spacing of the elements are too big [7]. To avoid grating lobes an element spacing of half the wavelength $(\lambda / 2)$ is necessary, and this applies for both the straight element and the interwoven. A $192+192$ RCA probe with a center frequency of $8 \mathrm{MHz}$ meets the requirement for the rodent experiment but is difficult to manufacture. The interwoven design is more flexible in the manufacturing process and therefore needs to be evaluated from an imaging perspective.

\section{B. Imaging sequence}

Synthetic Aperture imaging (SAI) have been widely used to enhance resolution and contrast in 2-D ultrasound imaging and increased frame rate [8]. For RCA SAI provides a method for insonifying an entire volume and collect backscattered signals from that region without being limited to a single plane. The simple SAI approach is to use a single element emission scheme where one single line element transmit at a time. All the $N$ orthogonal line elements are then active in receive and the data is beamformed through a delay-and-sum technique developed by Rasmussen et al [9]. The beamformed data for a single emission is referred to as a low resolution

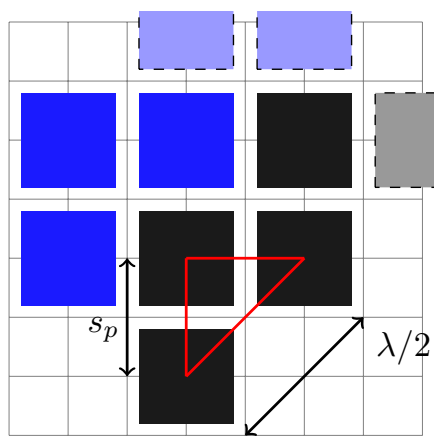

Fig. 2: Section of the interwoven structure, rotated 45 degrees. $s_{p}$ is the cell pitch. The red triangle displays the right-angle triangle used to estimate the cell pitch in eq. 1 for the zig zag structure. The intervowen structure have an cell spacing of $\lambda / \sqrt{8}$.

TABLE I: Parameters used in simulation

\begin{tabular}{l|c|}
\hline \multicolumn{2}{|c|}{ Straight } \\
\hline Number of cells $(M)$ & $192 \times 192$ \\
Number of elements/connections & $192+192$ \\
Center frequency & $8 \mathrm{MHz}$ \\
Wavelength $(\lambda)$ & $192.5 \mu \mathrm{m}$ \\
Line element pitch & $96.25 \mu \mathrm{m}$ \\
\hline \multicolumn{2}{|c|}{ Zig Zag structure } \\
\hline Number of cells $(M) \quad(2 \cdot 192) \times(2 \cdot 192)$ \\
Number of elements/connections & $192+192$ \\
Center frequency & $8 \mathrm{MHz}$ \\
Wavelength $(\lambda)$ & $192.5 \mu \mathrm{m}$ \\
Line element pitch & $96.25 \mu \mathrm{m}$ \\
Cell pitch $\left(s_{p}\right)$ & $68.05 \mu \mathrm{m}$ \\
\hline \multicolumn{2}{|c|}{192} \\
No. Transmit events & 2 cycles of a sinusiod \\
Emission Pulse type & $1540 \mathrm{~m} / \mathrm{s}$ \\
Roll of apodziation & pitch lengths of a Hanning \\
Speed of sound $(c)$ & parameters \\
\hline
\end{tabular}

volume (LRV), as there is no focusing in transmit. A new LRV is formed by emitting with the next line element in the transmit direction. Summing the contribution from all emissions reveals a high resolution volume (HRV), and the dynamic transmit focus is generated synthetically. This scheme is called single element RCA SAI.

The final HRV is comprised of $N$ transmit events corresponding to the number of elements in one of the two RCA dimensions. The transmit plane is referred to as the azimuthrange plane as steering in transmit is only performed using one side of the RCA array. The receive plane is referred to as the elevation-range plane. The delay-and-sum technique used here assumes that the time of flight is calculated as the distance from a point to a line, and the elongated row and column elements are assumed to be line elements. For the straight element RCA, the reference lines are defined as the center line of the element limited by the edges. For the interwoven design the acoustical center is assumed to be the geometric center line of the zig-zag element. This is visualized in Fig. 1. Here the green line symbolizes the row elements and column lines are marked with red. The zig zag structure will be further 

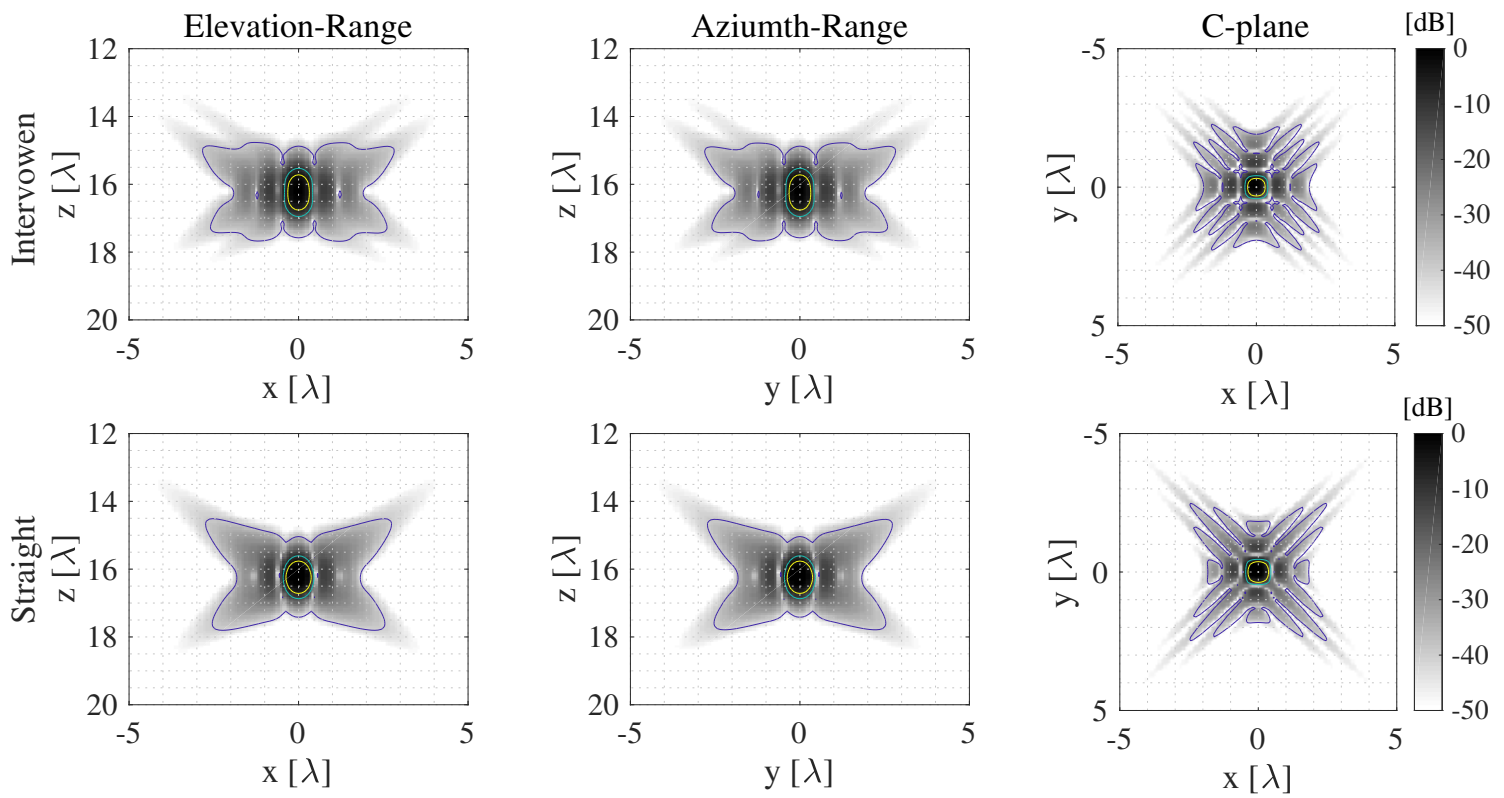

Fig. 3: Figure shows the range-elevation, range-azimuth, and elevation-azimuth planes beamformed from 192 emissions. Simulations parameters can be found in Table I. The top panel shows the results of the interwoven RCA beamformed planes and the bottom panel shows the same three planes for the straight RCA. The contour lines on top shows: $6 \mathrm{~dB}$ (yellow), 20 $\mathrm{dB}$ (green), and $40 \mathrm{~dB}$ (blue). The dynamic range is $50 \mathrm{~dB}$.

described in II-C.

\section{Implementation in Field II}

The straight RCA was implemented in Field II ([10], [11]) as $N$ row elements and $N$ column elements placed orthogonal to each other in space. The elements were implemented as elongated elements and the row aperture and column aperture was defined separately but spatially on top of each other. The interwoven structure was defined as the individual cells. The simulated received signals from the individual cells were summed without beamforming to emulate the expected responses from a zig-zag element. The line element pitch of the two models was the same for comparison reasons. The spacing in the zig-zag structure was determined by a rightangle triangle, shown in red in Fig. 2.:

$$
s_{p}^{2}+s_{p}^{2}=\left(\frac{\lambda}{2}\right)^{2} \quad \Leftrightarrow \quad s_{p}=\frac{\lambda}{\sqrt{8}} .
$$

Here $s_{p}$ is the cell pitch. To avoid ghost echos, integrated edge apodization was applied to the modelled as a drop in intensity corresponding to a Hanning weighted drop-off in intensity [9]. The parameters used can be found in Table I.

\section{Performance estimators}

A point spread function (PSF) was simulated for each of the two transducer models in Field II to estimate the performances. The quantifiable measures used were the fullwidth-half-maximum (FWHM) and the cystic radius at $20 \mathrm{~dB}$
TABLE II: Estimated metrics

\begin{tabular}{|l|ccc|}
\hline FWHM & Azimuth-Range & Elevation - Range & C-plane \\
\hline Straight & $0.62 \lambda$ & $0.60 \lambda$ & $0.60 \lambda$ \\
Interwoven & $0.61 \lambda$ & $0.62 \lambda$ & $0.62 \lambda$ \\
\hline CR20dB & Azimuth-Range & Elevation-Range & C-plane \\
\hline Straight & $1.42 \lambda$ & $1.42 \lambda$ & $1.43 \lambda$ \\
\cline { 1 - 3 } Interwoven & $1.34 \lambda$ & $1.34 \lambda$ & $1.30 \lambda$ \\
\hline
\end{tabular}

(CR20dB). Cystic resolution can be defined as [12]:

$$
C R(r)=\sqrt{\frac{E_{t 0}^{\text {out }}(r)}{E_{t 0}^{t o t}}}
$$

where $E^{\text {tot }}$ is the total PSF energy in an area and $E^{o u t}$ refers to the PSF energy outside the region of interest. In this case the region of interest is a growing sphere as a function of radius $r$. The reported value is the radius at which the $C R$ measure drops below $20 \mathrm{~dB}$. In case of a 3-D PSF, the ideal metric should be in 3-D. However such a measure have yet to be defined, so the two measures were calculated on the three orthogonal planes centered around the PSF. All metrics were normalized by the wavelength, as were the plots.

\section{RESULTS \& DISCUSSSION}

A simulation of a point scatter located at $[0,0,16.2] \lambda$ was performed for the two setups to extract the PSFs. The results are seen in Fig. 3. The two PSFs are showed using the same RCA SAI without apodization. Three orthogonal planes were beamformed and used to extract metrics to quantify performance. The metrics are found in Table II. The interwoven RCA showed similar resolution as to that of straight RCA with 


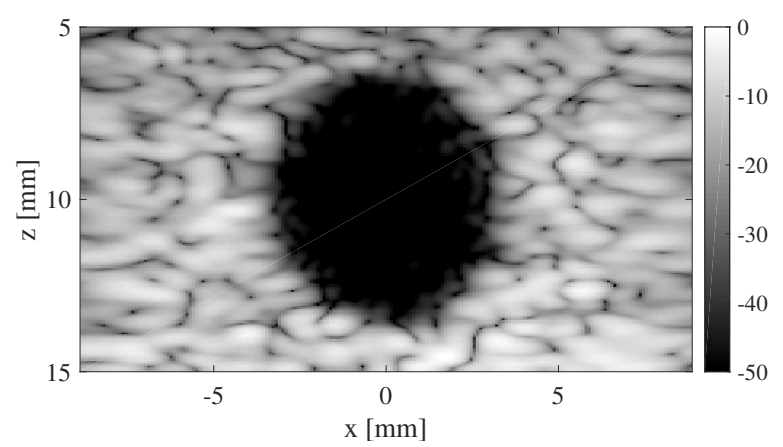

(a) Elevation-Range plane

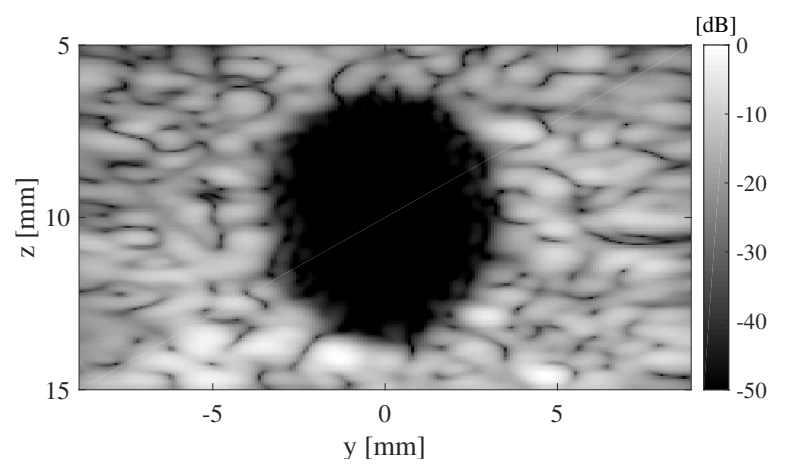

(b) Azimuth-Range plane

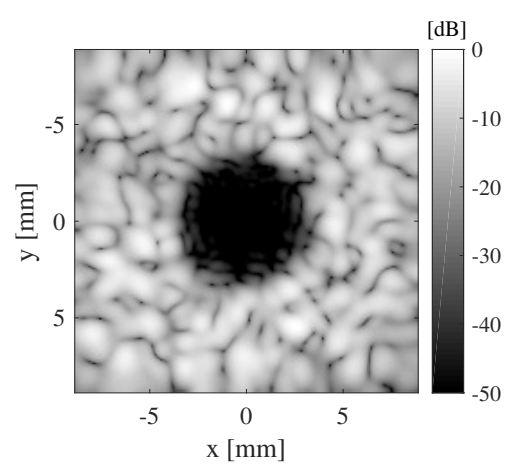

(c) C-plane at $10 \mathrm{~mm}$

Fig. 4: Three images shows a simulated cyst phantom, here imaged with SA single emission. The round shape of the cyst is maintained in both directions. a) shows the Elevation-Range plane, b) shows the Azimuth-Range plane and c) shows the C-plane at depth $10 \mathrm{~mm}$. All have a dynamic range of $50 \mathrm{~dB}$.

FWHM of approximately $0.61 \lambda$, which is close to the limit of the ideal diffraction limited resolution of $\lambda / 2$. The contrast quantified by CR20dB revealed a slightly better contrast for the straight RCA at 1.34 over $1.42 \lambda$. The PSFs showed symmetry in both the Azimuth-Range and Elevation-Range plot suggesting a symmetric geometry of the PSF, which is ideal for 3-D imaging. A spherical cyst phantom was simulated using zig-zag structured RCA model, with an optimized RCA SAI scheme. The cyst phantom consisted of a collection of scatters in a $10 \times 10 \times 10 \mathrm{~mm}^{3}$ region, a region corresponding to a sphere of radius $3 \mathrm{~mm}$ and a center of $[0,0,10] \mathrm{mm}$. The result can be seen in Fig.4.

\section{Closing Statement}

Simulations showed similar imaging performance for the interwoven and the straight RCA, close to the theoretical ideal FWHM of $\lambda / 2$. This proves that interwoven RCA design, from an imaging perspective, can be implemented instead of the straight one. The interwoven design allows for more flexibility in the manufacturing process. Having a $8 \mathrm{MHz}, 192+192 \mathrm{RCA}$ array can provide constant resolution in both directions of the 3-D volumes, which is ideal for SRI. It also allows for more advanced RCA SAI imaging schemes in the future.

\section{ACKNOWLEDGMENT}

This work was financially supported by the Danish National Advanced Technology Foundation (82-2012-4), Innovation Fund Denmark (7050-00004B) and by BK Medical, Herlev, Denmark.

\section{REFERENCES}

[1] C. Errico, J. Pierre, S. Pezet, Y. Desailly, Z. Lenkei, O. Couture, and M. Tanter, "Ultrafast ultrasound localization microscopy for deep superresolution vascular imaging," Nature, vol. 527, pp. 499-502, November 2015.

[2] K. Christensen-Jeffries, S. Harput, J. Brown, P. N. T. Wells, P. Aljabar, C. Dunsby, M. Tang, and R. J. Eckersley, "Microbubble axial localization errors in ultrasound super-resolution imaging," IEEE Trans. Ultrason., Ferroelec., Freq. Contr., vol. 64, no. 11, pp. 1644-1654, 2017.

[3] K. Christensen-Jeffries, R. J. Browning, M. Tang, C. Dunsby, and R. J. Eckersley, "In vivo acoustic super-resolution and super-resolved velocity mapping using microbubbles," IEEE Trans. Med. Imag., vol. 34, no. 2, pp. 433-440, February 2015.

[4] C. E. Morton and G. R. Lockwood, "Theoretical assessment of a crossed electrode 2-D array for 3-D imaging," in Proc. IEEE Ultrason. Symp., 2003, pp. 968-971.

[5] U. lok and P. C. Li, "Microbeamforming with error compensation," IEEE Trans. Ultrason., Ferroelec., Freq. Contr., vol. 65, no. 7, pp. 1153-1165, July 2018.

[6] K. B. Hansen, C. A. Villagomez-Hoyos, J. Brasen, K. Diamantis, V. Sboros, C. M. Sørensen, and J. A. Jensen, "Robust microbubble tracking for super resolution imaging in ultrasound," in Proc. IEEE Ultrason. Symp., 2016, pp. 1-4.

[7] M. F. Rasmussen and J. A. Jensen, "3-D ultrasound imaging performance of a row-column addressed 2-D array transducer: A measurement study," in Proc. IEEE Ultrason. Symp., July 2013, pp. 1460-1463.

[8] J. A. Jensen, S. Nikolov, K. L. Gammelmark, and M. H. Pedersen, "Synthetic aperture ultrasound imaging," Ultrasonics, vol. 44, pp. e5e15, 2006.

[9] M. F. Rasmussen, T. L. Christiansen, E. V. Thomsen, and J. A. Jensen, "3-D imaging using row-column-addressed arrays with integrated apodization - Part I: Apodization design and line element beamforming," IEEE Trans. Ultrason., Ferroelec., Freq. Contr., vol. 62, no. 5, pp. 947-958, 2015.

[10] J. A. Jensen, "Field: A program for simulating ultrasound systems," Med. Biol. Eng. Comp., vol. 10th Nordic-Baltic Conference on Biomedical Imaging, Vol. 4, Supplement 1, Part 1, pp. 351-353, 1996.

[11] J. A. Jensen and N. B. Svendsen, "Calculation of pressure fields from arbitrarily shaped, apodized, and excited ultrasound transducers," IEEE Trans. Ultrason., Ferroelec., Freq. Contr., vol. 39, pp. 262-267, 1992.

[12] K. Ranganathan and W. F. Walker, "Cystic resolution: A performance metric for ultrasound imaging systems," IEEE Trans. Ultrason., Ferroelec., Freq. Contr., vol. 54, no. 4, pp. 782-792, 2007. 